\title{
Bosminopsis brandorffi n.sp. (Crustacea, Cladocera) une nouvelle espèce de Bosminidae des systèmes Amazone et Orénoque
}

\author{
J. Rey ${ }^{1}$ \\ E. Vásquez ${ }^{2}$
}

Mots clés : Bosminopsis, nouvelle espèce, systèmes Amazone-Orénoque.

Bosminopsis brandorffi, nouveau Bosminidac, se différencie de toutes les autres espèces du genre par l'importance de la région céphalique, l'absence de rostre, le grand développement de l'œil et des antennules, la présence d'un fort talon abdominal. Mâles et femelles ephippiales sont inconnus.

Bosminopsis brandorffi n.sp. (Crustacea, Cladocera), a new species of Bosminidae from the Amazon and Orinoco river systems.

Keywords : Bosminopsis, Cladocera, new species, Amazon-Orinoco systems.

Bosminopsis brandorffi, a new species in the Bosminidae, is separated from all other species of the genus by features of the cephalic region, the lack of a rostrum, the well-developed eye and antennules, and the presence of a stronglydeveloped abdominal talon. Males and ephippial females are unknown.

Dans un travail analysant la structure des communautés de Rotifères et de Crustacés du Rio Nhamundá, G. O. Brandorff (1982) mentionnait, parmi d'autres formes nouvelles, la présence d'un Bosminopsis (Bosminopsis sp.) et le figurait brièvement. Ce nouveau Bosminidae s'avérait être également présent dans de nombreux autres sites : Rio Negro et ses affluents, Rio Aripuaña.

Presque dans le même temps, une étude de la composition longitudinale du zooplancton de l'Orénoque (Vásquez \& Rey 1989) révélait une forme identique dans les eaux vénézuéliennes. L'examen de ces exemplaires complétant le matériel amazonien nous a permis d'étudier cette nouvelle forme de Bosminopsis que nous dédions à Gert $\mathrm{O}$. Brandorff.

Bosminopsis brandorffi, n.sp.

\section{MATERIEL EXAMINE}

a) Amazonie (Brésil).

1. 65 spécimens collectés le 07.02.1963, Rio Negro, près de Tapuruquara. Leg $D^{r}$. H. Sioli.

1. Laboratoire d'Hydrobiologie, URA. 695 CNRS, Université P. Sabatier, 118 route de Narbonne, 31062 Toulouse Cedex.

2. Fundacion La Salle de Ciencias Naturales, Estación Hidrobiológica de Guayana, Apdo 51, San Fëlix, Edo Bolivar, Venezuela.
2. 2 spécimens collectés le 28.09.1974, réf. GOB-88-74, Rio Negro, près Itha Grande. Leg Dr Schaller.

3. 33 spécimens collectés le 25.09.1974, réf. GOB-83-74, Rio Negro. Leg Dr Schaller.

4. 32 spécimens collectés le 05.07.1975, réf. GOB-19-75, Rio Nhamundá, site 9 (cf. Brandorff et al. 1982). Leg. Dr G.O. Brandorff.

5. 3 spécimens collectés le 07.07.1975, réf. GOB-24-75, Rio Nhamundá, site 14. Leg Dr G.O. Brandorff.

6. 1 spécimen collecté le 04.06.1975, réf. RTAM-155-75, Rio Negro près Manaus. Leg Dr G.O. Brandorff.

b) Orénoque (Vénézuela)

Tous les individus collectés ont été récoltés en période de hautes eaux, du 18 au 25 septembre 1985 , en divers sites du fleuve Orénoque (cf. Vásquez \& Rey, 1989, p. 109).

1. 3 spécimens collectés site 1 , près de Samariapo.

2. 1 spécimen collecté site 2, près de PuertoAyacucho.

3. 2 spécimens collectés site 3 , près de El Burro.

4. 3 spécimens collectés site 7 , près de Ciudad Bolivar. 
La totalité du matériel étudié était constituée de femelles parthénogénétiques juvéniles et mâtures $\left(260_{\mu}\right.$ à $\left.480_{\mu}\right)$, dont seulement 2 femelles ovigères, chacune portant 3 œufs dans la cavité incubatrice. Aucun mâle ni femelle éphippiale n'a été observé.

Holotype : une femelle parthénogénétique mâture (réf. NE-SI-63a), $0.48 \mathrm{~mm}$ de longueur, conservée en tube dans une solution de formol à $4 \%$ additionnée de glycérine.

Paratypes : 7 femelles parthénogénétiques mâtures $(6$ individus conservés en tube dans une solution de formol à $4 \%$ additionnée de glycérine ; 1 individu monté à la glycérine), dont :

3 spécimens provenant du Rio Negro, près Tapuruquara (NE-HS-1-63, NE-HS-2-63, NEHS-3-63) ;

1 spécimen provenant du Rio Nhamundá (NHGOB-19-75) ;

2 spécimens provenant de l'Orénoque, près Samariapo (1 CHE-OR-SA-85, 1 BHE-ORSA-85) ;

1 spécimen provenant de l'Orénoque près Puerto-Ayacucho (2 bHE-OR-PA-85).

L'holotype et 4 paratypes sont déposés à l'Instituto Nacional de Pesquisas da Amazônia, Manaus (Brésil).

\section{Description}

Corps allongé, presque 2 fois plus long que large (fig. 1-2). La région céphalique est beaucoup plus développée que chez les autres espèces du genre : rapport hauteur tête/hauteur valves égal ou légèrement supérieur à 1 . Le bord dorsal présente un sinus cervical marqué. Les valves, cordiformes, sont arrondies à 1'angle antéro-ventral bordé de 10 soies pennées de taille décroissante vers l'arrière (fig. 3).

La tête (fig. 4) est large et dépourvue de rostre. A l'encontre des autres espèces du genre, la région antéro-frontale, fortement arrondie, est entièrement occupée par la cavité oculaire. L'œil, très volumineux, présente de nombreuses lentilles cristallines bien séparées du pigment. Le labre de grande taille est allongé, subrectangulaire, arrondi à son extrêmité garnie de cils.

Les antennules (fig. 1, 2,4) sont très développées. La région basale soudée est légèrement coudée à son départ de la tête. Sa longueur est 3 fois supérieure à celle de la partie distale, bifide, et garnie de spinules (fig. 5). Chacune des branches divergentes porte 8 longues papilles sensorielles à son extrêmité (fig. 6). Les 2 soies sensorielles antennulaires s'insèrent distalement aux $3 / 4$ environ de la partie basale commune.

Antennes. Le basipode est robuste, plus de 2 fois la longueur des branches antennaires (fig. 7). Il présente dans sa région basale articulaire 2 soies, l'une allongée et bisegmentée, l'autre courte et spiniforme, toutes deux insérées sur un petit tubercule (fig. 8). L'endopode, nettement plus court que l'exopode, porte de fines rangées de cils au niveau des $2^{e}$ et $3^{e}$ articles (fig. 9). Les soies antennaires, bisegmentées, sont longues : 1.5 fois la longueur de l'antenne. Formule antennaire : 0.0.3./1.1.3.

Post-abdomen. De forme conique, il se caractérise par l'existence d'un fort talon portant les 2 soies natatoires abdominales (fig. 1, 2, 10). Ce talon est très développé chez certains individus (fig. 11, 12). Les 2 soies natatoires abdominales, très longues, sont finement pennées à leur extrémité. La structure de la griffe terminale du post-abdomen (robuste et lisse), de même que celle de la griffe secondaire (plus courte), ne diffère guère de celle observée chez les autres espèces du genre. La dépression anale est bordée de cils courts et épais.

Patte thoracique I (fig. 13). La hampe est bien développée. L'endopode porte 4 soies d'inégale longueur réparties en un groupe de 3 soies bisétulées, dont 2 , beaucoup plus développées, présentent de longues sétules dans leur partie basale. La $4^{e}$ soie, la plus courte, s'insère à faible distance des précédentes dont elle est séparée par une légère protubérance finement ciliée. Exopode à 2 soies subégales, moyennement développées et bisétulées.

\section{Brève diagnose}

Corps allongé environ 2 fois plus long que large. Valves dépourvues d'épines. Région céphalique très développée. Rapport hauteur tête/hauteur valves égal ou légèrement supérieur à 1 . Oeil très volumineux, occupant toute la région céphalique antérofrontale fortement arrondie. Antennules très robustes à partie basale commune environ 3 fois la partie distale bifurquée. Post-abdomen à fort talon abdominal portant 2 très longues soies natatoires.

Si la structure des antennes et l'armature du postabdomen de Bosminopsis brandorffi rappellent les autres Bosminopsis, les caractères très originaux de la région céphalique (tête très large, rapport tête/valves $=1$, région antéro-f rontale fortement arrondie, 




Fig. 1-13. - Bosminopsis brandorffi, sp. nv. - Femelle parthénogénétique : 1-2. Vue latérale. 3. Détail de la région antéro-ventrale d'une valve. 4. Région antéro-frontale de la tête, antennules et labre. 5 . Région distale des antertnules. 6 . Papilles sensorielles de l'antennule. 7. Antenne : basipode et rames antennaires. 8. Soies sensorielles basales du basipode. 9. Région distale de l'endopode antennaire. 10-12. Post-abdomen. 13. Patte thoracique I. (Les échelles sont données en mm). 
œil très volumineux), de l'antennule (fort allongement de la partie basale commune) et du postabdomen (développement d'un fort talon abdominal), séparent d'emblée cette forme nouvelle et la situent très à part des 4 autres espèces du genre actuellement connues: Bosminopsis deitersi (Richard 1895), B. negrensis (Brandorff 1976), B. devendrai (Rane 1984), B. macaguensis (Rey \& Vásquez 1986).

Seule une étude comparative des structures mâles intéressant essentiellement les antennes, les antennules et la première paire d'appendices thoraciques, pourrait permettre de préciser des affinités interspécifiques.

\section{Distribution géographique}

a) Brésil : répartition établie par G.O. Brandorff (cf. $D^{r}$ B. Robertson, comm. pers.).

- Amazonie: Rio Negro, affluents du Rio Negro (Jauaperi, Jufaris), Rio Nhamundá.

- Mato Grosso: Rio Aripuanã (Cidade de Humbolt).

- Pará : Moju River, Curúa-Una Reservoir.

b) Vénézuela : Orénoque (Samariapo, PuertoAyacucho, El Burro, Ciudad Bolivar).

\section{Remerciements}

Nous exprimons tous nos remerciements à $\mathrm{D}^{\mathrm{r}}$ Barbara Robertson, INPA (Instituto Nacional de Pesquisas da Amazônia) Manaus, Brésil, qui nous a confié l'étude du matériel amazonien.

\section{Travaux cités}

Brandorff (G.O.). 1976. - A new species of Bosminopsis (Crustacea, Cladocera) from the Rio Negro. Acta Amazonica 6 (1) : $109-114$.

Brandorff (G.O.), Koste (W.) \& Smirnov (N.N.). 1982. - The composition and structure of Rotiferan and Crustacean communities of the Lower Rio Nhamunda, Amazonas, Brazil. Stud. Neotrop. Fauna and Envir. $17: 69-121$.

Rane (P.). 1984. - A new species of the genus Bosminopsis (Crustacea, Cladocera, Bosminidae) from India. J. Bombay Nat. Hist. Soc. 81 (3) : 668-669.

Richard (J.) 1895. - Description d'un nouveau Cladocère, Bosminopsis deitersi, n. gen., n. sp. Bull, Soc. Zool. Fr, 20 : 96.98.

Rey (J.) \& Vásquez (E.). 1986. - Bosminopsis macaguensis n. sp. et Alona ovata n. sp. (Crustacea, Cladocera), Cladocères nouveaux du Venezuela. Annis Limnol. 22 (3) : 219-229.

Vásquez (E.) \& Rey (J.). 1989. - A longitudinal study of zooplankton along the Lower Orinoco River and its Delta (Venezuela). Annis Limnol. 25 (2) : 107-120. 